\title{
Predictive and Prognostic Value of an microRNA Signature for Gastric Carcinoma Undergoing Adjuvant Chemotherapy
}

\section{Xiaowei Cheng}

Affiliated Hospital of Jiangnan University

Hongkuang Wei

The Maternal and Child Healthy Hospital of Guangxi Zhuang Autonomous Region

Sheng Zhang

Wuxi No. 8 People's Hospital

Fuzheng Zhang ( $\nabla$ wxsyzfz@163.com )

Affiliated Hospital of Jiangnan University https://orcid.org/0000-0001-6232-8464

\section{Research}

Keywords: Gastric carcinoma, Chemoresistance, microRNA, Predictive model

Posted Date: September 4th, 2020

DOI: https://doi.org/10.21203/rs.3.rs-70005/v1

License: (c) (i) This work is licensed under a Creative Commons Attribution 4.0 International License. Read Full License

Version of Record: A version of this preprint was published at DNA and Cell Biology on November 1st, 2021. See the published version at https://doi.org/10.1089/dna.2021.0377. 


\section{Abstract}

\section{Purpose}

Gastric carcinoma (GC) is one of the most common cause of tumor-related death. Chemotherapy resistance usually occurs, leading to cancer relapse and poor survival of GC patients. To investigate the role of miRNAs in chemotherapy resistance for GC patients, we conducted an integrated analysis of miRNA expression and survival information using data obtained from the TCGA project.

\section{Materials and methods}

Genome-wide screening of chemotherapy response-specific miRNAs was performed using Cox proportional hazards regression analyses for patients received chemotherapy or those had never received chemotherapy, respectively. CCK-8 assay was used for assessing cell viability. GSEA and single sample GSVA were performed for functional enrichment analysis. CIBERSORT cellular composition analysis was used to infer immune cell populations in GC tumors.

\section{Results}

A four-miRNA expression signature involving miR-130, -152, -199 and $-200 \mathrm{~b}$ was predicted as a specific indicator for $\mathrm{GC}$ chemoresistance $(P=0.00053$; hazard ratio $=8.63)$, outperforming those clinicopathological factors. Functional experiments confirmed the roles of these signature miRNAs in regulation of chemotherapy response. Functional enrichment of these signature miRNAs and risk score revealed positive association with epithelial-mesenchymal transition (EMT), and negative association with cell cycle checkpoint and DNA damage response. Furthermore, the immune infiltration-miRNA functional network analysis revealed transformation from activated effector cells to resting immunosuppressive cells are preferred in GCs with adverse chemotherapy response.

\section{Conclusions}

Our work identifies a four-miRNA expression signature as a promising chemoresistance biomarker in gastric carcinoma, which provides novel insights into developing new strategies to overcome GC chemoresistance.

\section{Background}

Gastric carcinoma (GC) is one of the most common causes of cancer-related deaths worldwide[1]. Although surgical removal is still the most effective way to treat gastric carcinoma, many GC patients have progressed to an advanced stage or have inoperable disease when they are diagnosed. Randomized trials have now established chemotherapy as standard adjuvant therapies[2]. There remain, however, significant divergence of clinical outcomes for GCs receiving adjuvant therapy[3]. Thus, identification of prognostic biomarkers is critical for guiding further treatment. 
microRNAs (miRNAs) are a family of small ( $22 \mathrm{nt})$ non-coding RNAs that function as posttranscriptional regulators of gene expression[4]. MiRNAs bind the 3'untranslated region (UTR) of the target mRNAs by base pairing, resulting in degradation and translation repression of mRNAs[4]. Increased studies have provide sufficient evidence revealing that miRNAs not only play crucial roles in normal developmental events, but also are widely involved in the development and progression of cancer types[5] through regulating expression of cancer-related genes implicated in cancer stem cell (CSC) maintenance, cancer cell metastasis and drug resistance[6,7]. However, our understanding on the roles of miRNAs in chemotherapy response remains limited.

There are many miRNAs that have been identified as oncogenic molecules or tumor suppressor[8]. Some miRNAs are very stable so that they could persist in peripheral blood. Therefore, numerous studies focused on miRNAs to discuss their relationship to both cancer cell biology and clinical practice. MiRNA profiling screenings have also been performed in various types of cancers including GC to identify prognosis-associated miRNAs as biomarkers to predict outcomes in patients. Chemotherapy resistance is the main obstacle to improve the survival rate of gastric cancer patients[9]. Several groups investigated in the role of miRNAs in drug resistance of gastric cancer, and revealed that high expression of some miRNAs like let-7g and miRNA-200c indicated sensitivity to chemotherapy[10]'[11]. In addition, some studies have also been carried out on the prediction of chemotherapy response by specific miRNA expression signatures. However, most of the studies focused on prognosis-related single miRNA or signature, the relationship between miRNA expression pattern and adjuvant chemotherapy was unexplored. Therefore, whether these mentioned miRNAs can be used to predict adjuvant chemotherapy response remains largely unknown.

We speculated that the difference of molecular biomarker expression pattern may represent the cause of prognosis variation in patients undergoing chemotherapy. Thus, our study aimed to identify and verify the miRNA biomarker that predict the chemotherapy response of GC patients using an integrated method. In this study, we performed an integrative analysis of whole-genome miRNA expression using data from The Cancer Genome Atlas (TCGA). We discovered a 4-miRNA signature-based risk scoring model to predict the adjuvant chemotherapy response of GC patients, which markedly outperform the clinical variables.

\section{Materials And Methods}

\section{Patients and omics data}

A total of $384 \mathrm{GC}$ patients was included in the TCGA GC cohort. The miRNA expression datasets of the TCGA gastric cancer cohort were obtained from the by using UCSC Xena browser (https://xenabrowser.net/). The clinical information and chemotherapy information were also downloaded from the TCGA dataset. The reads per million (RPM) mapped to each mature miRNA was considered as the expression level. Those miRNAs with RPM less than 0 in at least $50 \%$ of samples were discarded. Finally, we obtained a total of 544 miRNAs survived in the filtering process and subjected them to $\log _{2}$-transformation for subsequent analyses. 


\section{Identification of prognosis-related miRNAs specific for GC patients under adjuvant chemotherapy}

To identify prognosis-related miRNAs in the TCGA GC cohort, we first performed univariate Cox proportional hazards regression analysis for each miRNA based on groups (high- and low-expression groups) according to the median value of the expression levels in the patients with or without adjuvant chemotherapy. Then, those miRNAs with significant prognostic values in patients under adjuvant chemotherapy, but not patients without adjuvant chemotherapy, were subjected to construction of predictive model by using multivariate Cox proportional hazards regression analysis.

\section{Construction of prognosis-associated signature and nomogram}

Least absolute shrinkage and selection operator (LASSO), a parameter selection method that manage high-dimensional regression variables with no prior feature selection step by shrinking all regression coefficients and forcing many variables to be exactly zero, was used to select the optimal miRNAs for predictive model construction. The penalty regularization parameter I was determined by the 10 -fold cross-validation by using R package "glmnet"[12]. Finally, on the basis of the miRNA expression weighted by the coefficients generated by LASSO penalized regression, a four-miRNA signature was identified with the most minimum lambda value. The risk score for each patient was calculated as: Score $=L 1 \times$ Exp $1+$ L2×Exp2 +... LnxExpn, where Expi indicates the expression levels of the miRNAs and Li indicates the LASSO coefficients. Time-dependent receiver operating characteristic (ROC) curve (survivalROC [13] package in the R platform) was used to assess the predictive accuracy of the prognosis-associated signature. The "rms" package in the $\mathrm{R}$ was used to construct the nomogram based on the clinical parameters and the four-miRNA prognostic risk score in GC patients under adjuvant chemotherapy.

\section{Cell lines}

Briefly, eight GC cell lines, MKN45, MKN28, SNU16, SNU5, SNU1, HGC27, and AGS, and a human gastric mucosal cell, GES-1, were all commercially obtained from the American Type Culture Collection (ATCC). All cells were maintained in complete Dulbecco's Modified Eagle's medium (DMEM, Thermo Fisher Scientific, USA) containing 10\% fetal bovine serum (FBS, Thermo Fisher Scientific, USA), 100 units/ml penicillin, and 100 units $/ \mathrm{ml}$ streptomycin under $5 \% \mathrm{CO}_{2}$ in a humidified atmosphere at $37^{\circ} \mathrm{C}$.

\section{Quantitative Real-Time PCR (qRT-PCR) analysis}

TRIzol (Thermo Fisher Scientific, USA) was used to extract total RNA according to the manufacturer. The extracted total RNA was screened for purity and concentration was screened in a NanoDrop ${ }^{\text {TM }}$ One (Thermo Fisher Scientific, USA). 1 ug total RNA of indicated sample was used to synthesize cDNA with miScript II RT kit (Qiagen, Germany). To determine the expression of miR-199b, miR-152, miR-200b and miR-103a, SYBR Green PCR master mix (Takara, Janpan) was used and real-time PCR reactions were conducted in triplicate using StepOnePlus Real-Time PCR System (ABI, USA). The U6 was used as endogenous controls. 


\section{Proliferation assay}

Proliferation assays of GC cells were performed using 3-(4,5-dimethylthiazol-2-yl)-2,5-diphenyltetrazolium bromide (MTT) reagent following the instructions. Briefly, GC cells used for MTT assays were plated at a density of $5 \times 10^{3}$ cells/well in a 96-well plate in $200 \mu \mathrm{l}$ of complete DMEM medium per well. Between 24$120 \mathrm{~h}$ of incubation, $25 \mu \mathrm{l} \mathrm{of} 4 \mu \mathrm{g} / \mu \mathrm{l} \mathrm{MTT}$ (Sigma, USA) was added to each well at a final concentration of $0.5 \mu \mathrm{g} / \mu \mathrm{l}$. After a $2-4 \mathrm{~h}$ incubation at $37^{\circ} \mathrm{C}$, the culture medium was gently aspirated, $120 \mu$ dimethyl sulfoxide (DMSO) was added to dissolve the resulting MTT formazan crystals. The optical density (OD) value of each well was examined at absorbance of $570 \mathrm{~nm}$ via the enzyme-linked immunosorbent assay (ELISA) microplate reader (Bio-Rad, USA) to measure the relative cell proliferating rate.

\section{5-Fluorouracil (5-FU) assay}

The AGS and MKN28 cells were incubated with 5-Fluorouracil for $24 \mathrm{~h}$ (Sigma, USA) at concentrations of $0,0.31,0.63,1.25,2.5 \mu \mathrm{g} / \mathrm{ml}$. The cell viability was determined by MTT assays. Each assay was conducted in triplicate, and the data was normalized against the OD570 value on that of control well to measure the relative viability of each well.

\section{Overexpression of miRNAs}

The MKN28 and AGS cells were harvested and seeded into a 6-well plate at the concentration of $2 \times 10^{5}$ cells/well in complete DMEM medium. Then the cells were transfected with either miR-199b, miR-152, miR-200b, miR-103a mimics or mimic negative control (mimic-Ctrl) at the final concentration of $2.5 \mu \mathrm{M}$ with Lipofectamine 3000 (Thermo Fisher Scientific, USA). All the miRNA and negative control mimics were purchased from Ribobio (Guangzhou city). The sequences used for each miRNA used are described below: miR-199b mimic, CCCAGUGUUUAGACUAUCUGUUC; miR-152 mimic, UCAGUGCAUGACAGAACUUGG; miR-200b mimic, UAAUACUGCCUGGUAAUGAUGA; miR-103a mimic, AGCAGCAUUGUACAGGGCUAUGA; mimic-Ctrl, AGUGCAUGUUAUGCCUACG.

\section{Prediction of miRNA targets, $\mathrm{GO}$ and pathway enrichment analysis}

Candidate target genes of indicated miRNAs were predicted using two different online databases, including TargetScan [14] and StarBase [15]. Candidate miRNA target genes which were repeatedly predicted by both databases were retained for further functional enrichment analysis. We used Database for Annotation, Visualization, and Integrated Discovery (DAVID)[16] to perform Gene Ontology (GO) enrichment analysis and Kyoto Encyclopedia of Genes and Genomes (KEGG) analysis on the hypomethylation-high expression putative target genes.

\section{Gene set enrichment analysis (GSEA)}

GSEA analysis with miRNA target gene sets was carried out by GSEA software (version 4.0)[17] with Molecular Signature Database (MSigDB) HALLMARK collection. A weighted enrichment statistic was 
used. Phenotype permutations were performed on samples with a permutation number of 1000 , and terms with false discovery rate $(F D R)<0.1$ were considered to be significantly enriched.

\section{Inference of infiltrating immune cells.}

The relative proportions of 22 types of infiltrating immune cells were inferred by using CIBERSORT (http://cibersort.stanford.edu/) [18] in transformed gene expression data from bulk tumors of GC. The 22 immune cell types include T cells, B cells, macrophages, natural killer cells, eosinophils, neutrophils, dendritic cells and amongst others. CIBERSORT infers immune cell type proportions with the signature matrix (containing 547 genes) as a reference, which represents the marker genes for each cell type, by using support vector regression [18]. The significance of deconvolution for each immune cell type was assessed by using Monte Carlo sampling with 1,000 permutations.

\section{Statistical analysis}

The $\chi 2$ test was used to compare the correlations of continuous and categorical variables between the patients received with and without chemotherapy. Univariate proportional hazards regression analyses were used to determine the independent prognostic variables for Overall survival (OS). To perform survival analysis, the Kaplan-Meier method and log-rank test was used to generate and compare survival curves. The time-dependent receiver operating curve (ROC) was carried out to assess the predictive accuracy and sensitivity of each variable and the 4-miRNA signature. The activity of pathway was inferred by single sample GSVA [19]. A threshold of $P<0.05$ was determined as statistically significant. R version 3.4.4 were applied to the statistical analyses.

\section{Data Availability Statement}

The data that support the findings of this study are available from TCGA. Restrictions apply to the availability of these data, which were used under license for this study. Data are available at https://portal.gdc.cancer.gov/ with the permission of TCGA.

\section{Results}

\section{Clinical significance of chemotherapy in GC patients}

A total of $384 \mathrm{GC}$ patients was included in the TCGA GC cohort. Among the $384 \mathrm{GC}$ patients, $48.7 \%$ (187/384) had received adjuvant chemotherapy (GC-ACT), while 50.5\% (194/384) didn't receive any types of chemotherapy (GC-NOACT), (Figure 1A). Clinical and pathological characteristics of these patients are summarized in the Table S1. Patients were well balanced for gender $(P>0.05)$. Also, no statistically significant difference was observed according to primary lymph node, pathologic $\mathrm{T}, \mathrm{N}$ and $\mathrm{M}$ stages, and histologic grade. Adjuvant chemotherapy administration for each type of drugs were summarized as shown in Figure 1A. The most used chemotherapy drug for GC patients is Fluorouracil (61/187, 32.6\%). Almost half of the GCs received chemotherapy gain beneficial from chemotherapy (Figure 1B). Of note, $45.5 \%$ GCs exhibited complete response (CR) to adjuvant chemotherapy (Figure 1B). Kaplan-Meier 
survival curves showed that the hazard ratio (HR) for overall survival among those who didn't receive adjuvant chemotherapy was 2.14 (95\% confidence interval $[\mathrm{Cl}]=1.32-3.49, \mathrm{P}=0.0022$; Figure 1C). Taken together, these results indicated that the adjuvant chemotherapy is an actionable treatment strategy for GC patients.

\section{Identification of specific prognostic biomarkers in GC patients undergoing chemotherapy}

Given the marked effect of chemotherapy for GC patients, we thus wondered whether there are prognostic biomarkers for GCs those received adjuvant chemotherapy. As a type of widely used biomarker, miRNAs exhibited good performance for cancer diagnosis and prognosis. We obtained the miRNA expression data from the TCGA GC cohort. After quality control, a total of 544 miRNAs were subjected for further analyses. To explore prognosis-associated miRNAs specific for GC patients received chemotherapy OS, but not for GC patients not received chemotherapy, we conducted Cox proportional hazards regression analysis for each miRNA in GC-ACT and GC-NOACT patients, respectively. miRNAs $(n=63)$ those with $P<$ 0.05 in GC-ACT and P > 0.05 in GC-NOACT were remained.

Then, the LASSO Cox regression analysis was conducted on 63 candidates to identify robust markers. By forcing the sum of the absolute value of the regression coefficients under a fixed value, certain coefficients were shrunk to exactly zero, and the most powerful prognostic markers were identified with relative regression coefficients. Cross-validation was applied to prevent the over-fitting of the LASSO Cox model (Figures 2A and 2B). Four miRNAs were finally identified as independent prognostic biomarkers for GC-ACT. These four miRNAs included miR-199b, miR-152, miR-200b and miR-103a.

\section{Prognostic value of a four-miRNA signature risk score in GC undergoing chemotherapy}

To assess the prognostic value of miR-199b, miR-152, miR-200b and miR-103a, we constructed a prognostic signature by integrating the expression of these four miRNAs using the regression coefficients. The risk-score formula was as followings: risk score $=\left(5.064341 \mathrm{e}-05 \times \operatorname{Exp}_{\text {miR-199b }}\right)+(4.946951 \mathrm{e}-04 \times$ $\left.\operatorname{Exp}_{\text {miR-152 }}\right)+\left(-2.764529 \mathrm{e}-05 \times \operatorname{Exp}_{\mathrm{miR}-200 \mathrm{~b}}\right)+\left(-4.906671 \mathrm{e}-05 \times \operatorname{Exp}_{\mathrm{miR}-103 \mathrm{a}}\right)$. We calculated the risk scores of GC-ACT patients and GC-NOACT patients using above formula, respectively. The ranked risk scores of patients in each sample set was showed as Figures 2C and 2D. Heatmaps described the expression profiles of these four miRNAs in sample set, and the samples were ranked according to their risk scores (Figures 2C and 2D). Among the four miRNAs, miR-199b and miR-152 received a positive coefficient and acted as risk factors. The other two miRNAs with negative coefficients, including miR-200b and miR-103a, acted as protective factors.

To confirm the specific prognostic value of these four miRNAs for GC patients received adjuvant chemotherapy, we examined the association of each miRNAs with OS in GC-ACT and GC-NOACT patients, respectively. As expected, Kaplan-Meier survival curves showed that high expression levels of miR-199b and miR-152 were associated with poor survival of GC-ACT patients, while high expression levels of miR$200 \mathrm{~b}$ and miR-103a were associated with better survival of GC-ACT patients (Figure 2E). On the contrary, none of them displayed prognostic value for GC-NOACT patients (Figure 2F). 
We further assessed the prognostic value of the four-miRNA signature risk model. GC-ACT and GCNOACT patients were classified into a high-risk or a low risk group based on the median risk score, respectively. Kaplan-Meier analysis was used to evaluate the impact of the four-miRNA signature on the OS of GC-ACT and GC-NOACT patients, respectively. The results showed that the high-risk group had a significantly poorer OS than that of the low-risk group in GC-ACT patients (Figure $3 A$ ). We used timedependent ROC analysis to assess the prognostic significance of four-miRNA signature risk score. The areas under the ROC curve (AUC) for 1-, 3- and 5-year OS of GC-ACT patients were 0.777, 0.827 and 0.865, respectively, indicating a favorable prognostic value in predicting chemotherapy outcomes (Figure 3A). However, Kaplan-Meier analysis of the four-miRNA signature in GC-NOACT showed no significant association between the risk score and the OS of GC-NOACT patients (Figure 3B). Consistently, the AUCs for 1-, 3- and 5-year OS of GC-NOACT patients were 0.501, 0.595 and 0.595 , respectively, suggesting its low performance in GC-NOACT patients (Figure 3B). Taken together, these results indicated that the fourmiRNA signature may thus be useful as biomarkers for prediction of chemotherapy response in GCs.

To compare the prognostic value of miRNA-based predictor and other clinical variables for chemotherapy outcomes of GC patients, time-dependent ROC analysis was performed for 1-, 3- and 5-year survival, respectively. The clinical variables used for comparation include age, gender, number of lymphnodes, histologic grade, individual pathologic T, N and M stages and the combined pathologic stage. The result showed that the four-miRNA signature risk score outperformed all the clinical variables, especially for short-term (1-year and 3-year survival) survival prediction (Figures $\mathbf{3 C}$ and $\mathbf{3 D}$ ). There is no clinical variable exhibiting an AUC greater than 0.7 for 1 - and 3-year survival prediction (Figures $3 \mathrm{C}$ and $3 \mathrm{D}$ ). The most predictive variable is the pathologic $\mathrm{M}$ stage for 3 -year survival prediction (AUC $=0.623$ ). For longterm survival prediction, the histologic grade showed a relative greater performance with an AUC of 0.703 (Figure 3E). Taken together, the time-dependent ROC analyses revealed that all the predictors, either miRNA-based signature or other clinical variables, present better performance for long-term survival prediction than that for short-term survival prediction (Figure 3F). Of note, the four-miRNA signature outperformed other clinical variables.

\section{Functional roles of signature miRNAs in regulation of chemotherapy response}

To explored the functional role of these signature miRNAs in regulation of chemotherapy response in GC cells, we first examined the four-miRNA signature risk score in GC-ACT patients according to the clinical response. In agree with the Kaplan-Meier survival analysis result, GC-ACT patients who gain beneficial from adjuvant chemotherapy (CR and PR) have lower risk scores than GC-ACT patients with no response after chemotherapy (Figure 4A). Then, the expression levels of these four signature miRNAs were examined in GC cell lines. Analysis of qRT-PCR demonstrated that, as compared to non-carcinoma CES-1 cells, miR-199b and miR-152 expressions were significantly upregulated in GC cell lines of MKN45, MKN28, SNU16, SNU5, SNU1, HGC27 and AGS cells (Figure 4B). Conversely, miR-200b and miR-103a expressions were significantly downregulated in GC cell lines compared to GES-1 cells (Figure 4B). 
Next, we assessed the effects of overexpression of these signature miRNAs on the regulation of GC cell chemosensitivity to Fluorouracil. MKN28 cells, with low levels of endogenous miR-199b and miR-152, were transfected with miR-199b mimics or miR-152 mimics under the treatment with various concentrations of 5 -Fluorouracil for $24 \mathrm{~h}$. MTT assays showed overexpression of miR-199b or miR-152 significantly increased the chemoresistance of MKN28 cells to 5-Fluorouracil (Figure 4B). On the contrary, overexpression of miR-200b and miR-103a in AGS cells, with low levels of endogenous miR-200b and miR-103a, dramatically sensitized AGS cells to 5-Fluorouracil (Figure 4B). These observations are consistent with the prognostic role of each miRNA for GC-ACT patients: miR-199b or miR-152 as risk factors while miR-200b and miR-103a as protective factors. Taken together, our results indicated that these signature miRNAs may paly pivotal roles in modulation of chemotherapy efficiency.

\section{Construction of a nomogram to predict outcomes in GC patients undergoing chemotherapy}

To provide a clinically associated quantitative method that could be used to predict the probabilities of 1-, 3-, 5- and 10-year OS in GC patients received adjuvant chemotherapy, a prognostic nomogram was established in which the score integrated the clinical variables, including age, gender, number of lymphnodes, histologic grade, individual pathologic $\mathrm{T}, \mathrm{N}$ and $\mathrm{M}$ stages and the combined pathologic stage, and the four-miRNA signature (Figure 5A). The calibration curves for the nomogram for OS of GC patients received adjuvant chemotherapy showed good agreement between the prediction and the actual observation (Figure 5B). The AUC functions of the multivariable models were developed to indicate how well these features serve as prognostic markers. Compared to the clinical variables, the four-miRNA signature and the nomogram showed relatively high predictive power for OS of GC patients received adjuvant chemotherapy, with an average AUC above 0.844 and 0.832 , respectively (Figure 5B).

\section{Functional involvement of the risk signature miRNAs}

To evaluate the potential function of the four signature miRNAs, we inferred the activity of HALLMARK and KEGG pathways by gene set variation analysis (GSVA) method on the basis of single sample expression data [19]. By examining the correlation between the expression levels of risk signature miRNAs and the activity of each pathways documented by these two databases, we found that mir-199b and miR-152 showed similar pattern, which is contrast to the pattern in mir-200b and mir-103a-1. Briefly, high levels of mir-199b and miR-152 are negatively correlated with the cell cycle checkpoint, DNA damage response and RNA processing, and positively associated with myogenesis, epithelial-mesenchymal transition (EMT), MAPK and JAKA-STAT singling (Figure 6A and 6B). Conversely, mir-200b and mir-103a1 exhibited opposite associations (Figure 6A and 6B). Notably, no obvious difference of correlations was observed between GC patients with adjuvant chemotherapy and those without adjuvant chemotherapy (Figure 6A and 6B).

To investigated the functional involvement of the targets of risk signature miRNAs, we then predicted the putative targets using TargetScan and Starbase database, respectively. KEGG pathway analysis revealed that the target genes mainly enriched in metabolism (such as glycosphingolipid biosynthesis-lactoand neolacto series, tryptophan metabolism, amino sugar and nucleotide sugar metabolism), axon guidance 
and lysosome (Figure 6C and Table S2). G0 analysis included molecular function (MF), biological process (BP), and cellular component (CC). The target genes were mainly related with ion binding (MF), cellular nitrogen compound metabolic process (BP), organelle (CC) (Figure6C).

Further, to investigate the functional involvement of the four-miRNA risk score, we performed gene set enrichment analysis (GSEA) in the gene expression data from the TCGA GC-ACT patients. According to the median value of the risk score, we divided the patients into two groups: high- and low-risk group. GSEA results showed that the high-risk group is significantly associated with hallmarks involved in cancer metastasis, such as epithelial-mesenchymal transition (EMT) (FDR q $<0.1$; Figure 6E and Table S3). EMT has been considered as a pivotal contributor to drug resistance, but the mechanism is still elusive. Recent cancer stem cell (CSC)-associated studies provide a new insight into the mechanism of drug resistance in cells undergoing EMT [20]. On the contrary, cell cycle checkpoint-associated hallmarks were significantly enriched in the low-risk group, such as E2F targets and G2M checkpoint (FDR q < 0.1; Figure 6E and Table S4). It has been found that activation of cell cycle checkpoint is efficient type of anticancer therapeutic. Increasing number of studies are revealing that chemical manipulation of cycle checkpoint activation increases the killing efficacy of major cancer therapeutics as well as to directly promote cancer cell death [21].

\section{Aberration of immune cell infiltration correlates with chemotherapy response}

miRNAs have been identified as pivotal modulators in regulation of the development and the function of immune subpopulations involved in tumor immune response. Thus, to assess the contribution of miRNAs to immune infiltration in GC, we investigated the correlation between the expression levels of risk miRNAs and immune cell infiltration in GCs. The relative proportions of 22 types of infiltrating immune cells were inferred by using CIBERSORT (http://cibersort.stanford.edu/) [18] in transformed gene expression data from bulk tumors of GC (Figure 7A). The 22 immune cell types include T cells, B cells, macrophages, natural killer cells, eosinophils, neutrophils, dendritic cells and amongst others.

The miRNA-immune infiltration correlation analyses exhibited that mir-199b and miR-152 showed an opposite pattern to mir-200b and mir-103a-1 (Figure 7B). mir-199b and miR-152 with risk effects exhibited negative correlations with the infiltration of $\mathrm{T}$ follicular helper (Tfh) cells, plasma cells and activated dendritic cells (DCs), and positive correlations with the infiltration of monocytes, memory B cells and M2 macrophages (Figure 7B). On the contrary, mir-200b and mir-103a-1 with protective effects, displayed strong positive correlations with the infiltration of activated mast cells, Tfh cells, M0 macrophages, plasma cells and activated DCs, while marked negative correlations with the infiltration of resting mast cells, regulatory $\mathrm{T}$ (Treg) cells, memory B cells and resting DCs, Figure 7B). Among the correlates, mast cells exhibited the strongest effects with mir-200b and mir-103a-1 (Figure 7B). These data suggested that GCs with high risk to adjuvant chemotherapy are characterized with enrichment of resting suppressive immune cells, such as Treg, resting mast cells and resting DCs, while GCs those got beneficial from the adjuvant chemotherapy are dominated by effector immune cells, such as activated mast cells, Tfhs, plasma cells and activated DCs. 
Furthermore, to further explore the roles of these risk signature miRNAs in immune response, we examined the expression levels of each miRNA in the GCs according to the immune subtypes predicted by previous study [22]. GC samples were grouped into five immune subtypes: C1-wound healing, C2-IFN- $\gamma$ dominant, C3-inflammatory, C4-lymphocyte depleted and C6-TGF- $\beta$ dominant, characterized by differences in macrophage or lymphocyte signatures, Th1:Th2 cell ratio, extent of intratumoral heterogeneity, aneuploidy, extent of neoantigen load, overall cell proliferation, expression of immunomodulatory genes, and prognosis [22]. Interestingly, GCs in C6-TGF- $\beta$ dominant subtype have relative higher expression levels of mir-199b and miR-152 than GCs in the other subtypes (Figure 7C), especially in GCs with chemotherapy, consistent with the immunosuppressive role of TGF- $\beta$ signaling in cancers. On the contrary, mir-200b and mir-103a-1 showed relative higher expression levels in C1-wound healing and C2-IFN- $y$ dominant subtypes than that in the others, suggesting active immune responses

(Figure 7C). Taken together, these findings indicated that the risk signature miRNAs may act as immune response mediators in the regulation of chemotherapy response.

\section{Discussion}

Drug resistance to chemotherapy and molecular targeted therapy is an important problem in current cancer research[23]. Chemoresistance refers to the resistance of malignant cells to various chemotherapeutic agents with different molecular mechanisms and structures, which is the cause of recurrence and metastasis in most malignant tumors including GC. There is an urgent need to accurately identify the malignant status of tumors using novel biomarkers with prognostic significance, which will significantly assist the development of clinical precision medicine and ultimately improve the prognosis of tumor patients. The miRNA expression profile of drug resistant cancer cells is quite different from the drug sensitive parental cancer cells[24]. The aims of this study were to identify a miRNA signature as a potential prognostic marker for GC patients received adjuvant chemotherapy in the clinic.

We here conducted an integrative analysis of whole-genome miRNA expression. Using the multivariate Cox coefficients of LASSO analysis, we developed a 4-miRNA-based risk scoring signature for prediction adjuvant chemotherapy response of GC patients, which markedly outperform the clinical variables. Using our miRNA-based model, we also developed the nomogram, a scoring system ranging from 0 to 100 that can predict risk of death after adjuvant chemotherapy. Our findings strongly suggest that both the fourmiRNA model and nomogram provide independent values beyond conventional clinical variables.

Among the four signature miRNAs, two of them, including miR-199b and miR-152, acted as risk factors for GCs, and the other two miRNAs (miR-200b and miR-103a) were protective factors. Notably, these miRNAs have specific predictive value for GC-ACT patients, but not GC-NOACT patients. Thus, this type of miRNAs may exert pivotal role in modulation of chemotherapy response in GCs. miR-199b expression is upregulated in Wilms' tumor, and miR-199b can directly target RUNX3 to promote Wilms' tumor progression, indicating that the miR-199b/RUNX3 axis represents a potential target for Wilms' tumor treatment[25]. miR-199b also serve as an oncogene in osteosarcoma (OS). Upregulated miR-199b is significantly related to OS stage, distant metastasis and poor prognosis. This miRNA may promote 
progression of OS[26]. However, miR-199b has been reported to be under-expressed in several other types of cancer. For example, the expression level of miR-199b is down-regulated in hepatocellular carcinoma (HCC). HCC patients with low miR-199b expression levels show lower survival rates than those with high miR-199b expression levels[27,28]. In addition, down-regulation of miR-199b is widely observed in bladder cancer[29], breast cancer[30], colorectal cancer[31], acute myeloid leukemia[32], non-small cell lung cancer[33] and endometrial carcinoma[34]. The above contradictory findings suggest that the expression pattern of miR-199b is tissue-specific, and miR-119b may represent a new prognosis-associate biomarker. miR-152 has also been reported to be involved in the progression of multiple cancer types, both as oncogene and tumor suppressor gene. miR-152 is over-expressed in some types of cancer, including liver cancer[35], ovarian cancers[36], gastrointestinal cancers[37], as well as in lung cancers[38].The low plasma level of miR-152 significantly predict the poor survival of squamous cell carcinoma lung cancer patients[39]. On the other hand, several studies show that miR-152 may play tumor suppressor role in other types of cancers like bladder cancer[40]. miR-200b belongs to the miR-200 family, which is widely known for its anti-tumor function in a variety of cancers, including breast cancer[41], colorectal cancer[42], pancreatic cancer[43], endometrial carcinoma[44] and gastric cancer[45]. miR-200b and miR$200 \mathrm{c}$ have been revealed as potential prognostic bio-markers of gastric cancer[45]. With regard to the fourth miRNA, miR-103, increasing evidences have indicated that it acts as an oncogene in tumor development and progression[46]' [47]. However, the role of miR-103 in gastric cancer is controversial[48]' [49]' [50]. Although these miRNAs are known to play important roles in tumorigenesis, most of them have not been reported as prognostic biomarker for GC patients received adjuvant chemotherapy in the literature. Further researches should focus on the interaction between these miRNAs to help us develop optimal treatment strategy for GC patients undergoing adjuvant chemotherapy. This provides new ideas for the study of chemotherapy response machines in GC.

We also performed pathway enrichment analysis of the four signature miRNAs target genes to reveal the underlying molecular mechanisms. The mostly enriched pathways were involved in metabolism (such as glycosphingolipid biosynthesis-lacto and neolacto series, tryptophan metabolism, amino sugar and nucleotide sugar metabolism), axon guidance and lysosome, etc. It indicated that the four-miRNA might function as tumor metabolism modulator involved in chemotherapy response of GCs. Glycolipid metabolism and biology, including the glycosphingolipid composition of cell membrane and their transport and communication across the barriers, have provide new perspectives for tumorigenesis and drug resistance research. For instance, the expression levels of glucosylceramide and galactosyl ceramide in doxorubicin-resistant ovarian carcinoma (OC) cells are three times higher than those in doxorubicin-sensitive wild-type OC cells[51]. Besides, the expression levels of ganglioside, especially GM3 and GM2, in fenretinide-resistant $\mathrm{OC}$ cells are six times higher than those in fenretinide-sensitive $\mathrm{OC}$ cells[52]. Both established drug-resistant ovarian adenocarcinoma cell line and tumor samples from chemotherapy-resistant patients show increased glucosylceramide levels[53]. Thus, these findings together with our study provide a link between chemotherapy response-associated miRNAs and the glycosphingolipid biosynthesis. 
In addition to functional enrichment analysis of signature miRNA target genes, GSEA revealed that the 4miRNA signature identified high-risk group was positively related to EMT and apical junction, while negatively related to cell cycle transition, partly explaining the poor prognosis of GC patients with chemoresistance. Although EMT is widely considered to play a key role in tumor progression and metastasis, two recent studies link EMT to drug resistance in lung cancer and pancreatic cancer, respectively[54,55]. It's more and more clear that EMT is a sophisticated biological event regulated by a series of signaling pathways. Whether EMT represents the main cause of drug resistance in GC remains to be further studied.

There are still some limitations in our study. First, in this retrospective study, there is a lack of diverse ethnic groups in our cohort. Second, the predictive value of our four-miRNA model needs to be further validated in larger prospective cohort. Third, though we conducted in vitro experiments to assess the modulatory roles of those signature miRNAs in chemotherapy response in GC cells, further in-deep functional studies are needed.

In conclusion, the newly identified 4-miRNA signature was demonstrated as an effective and stable model to predict the prognosis of GC patients received adjuvant chemotherapy, which outperformed the clinicopathological features. The clinical application of this 4-miRNA signature will contribute to risk classification, thus guiding personalized therapy for GC patients. While systematic experimental verification is lacking, our research provides a basis for the use of miRNA panel as a clinical tool for prognostic assessment after adjuvant chemotherapy and these four miRNAs may also be potential therapeutic targets for the treatment of drug resistance in GC patients.

\section{Declarations}

\section{Ethics approval and consent to participate}

The study was approved by the Medical Ethics Committee of the Affiliated Hospital of Jiangnan University. These is no clinical samples recruited by us in the present study.

\section{Consent for publication}

Not available.

\section{Availability of data and materials}

The data were obtained from TCGA (https://portal.gdc.cancer.gov/) data portal.

\section{Competing interests}

All authors declare that he/she has no conflict of interest.

\section{Funding}


Not available.

\section{Authors' contributions}

FZZ designed the study. FZZ and XWC drafted and revised the manuscript. XWC performed bioinformatics analyses. HKW provided help for bioinformatics analyses. SZ performed functional experiments. XWC and HKW performed survival analysis and interpreted the data.

\section{Acknowledgements}

The authors thank the contributors of the TCGA (https://portal.gdc.cancer.gov/) for sharing their data on open access.

\section{References}

1. Rahman R, Asombang AW, Ibdah JA (2014) Characteristics of gastric cancer in Asia. World J Gastroenterol 20 (16):4483-4490. doi:10.3748/wjg.v20.i16.4483

2. Cunningham D, Allum WH, Stenning SP, Thompson JN, Van de Velde CJ, Nicolson M, Scarffe JH, Lofts FJ, Falk SJ, Iveson TJ, Smith DB, Langley RE, Verma M, Weeden S, Chua YJ, Participants MT (2006) Perioperative chemotherapy versus surgery alone for resectable gastroesophageal cancer. $\mathrm{N}$ Engl J Med 355 (1):11-20. doi:10.1056/NEJMoa055531

3. Marin JJ, Al-Abdulla R, Lozano E, Briz O, Bujanda L, Banales JM, Macias RI (2016) Mechanisms of Resistance to Chemotherapy in Gastric Cancer. Anticancer Agents Med Chem 16 (3):318-334. doi:10.2174/1871520615666150803125121

4. Lu J, Getz G, Miska EA, Alvarez-Saavedra E, Lamb J, Peck D, Sweet-Cordero A, Ebert BL, Mak RH, Ferrando AA, Downing JR, Jacks T, Horvitz HR, Golub TR (2005) MicroRNA expression profiles classify human cancers. Nature 435 (7043):834-838. doi:10.1038/nature03702

5. Lee YS, Dutta A (2009) MicroRNAs in cancer. Annual review of pathology 4:199-227. doi:10.1146/annurev.pathol.4.110807.092222

6. Lou W, Liu J, Gao Y, Zhong G, Chen D, Shen J, Bao C, Xu L, Pan J, Cheng J, Ding B, Fan W (2017) MicroRNAs in cancer metastasis and angiogenesis. Oncotarget 8 (70):115787-115802. doi:10.18632/oncotarget.23115

7. Garg M (2015) Emerging role of microRNAs in cancer stem cells: Implications in cancer therapy. World journal of stem cells 7 (8):1078-1089. doi:10.4252/wjsc.v7.i8.1078

8. Slack FJ, Chinnaiyan AM (2019) The Role of Non-coding RNAs in Oncology. Cell 179 (5):1033-1055. doi:10.1016/j.cell.2019.10.017

9. Linkous AG, Yazlovitskaya EM (2012) Novel radiosensitizing anticancer therapeutics. Anticancer Res 32 (7):2487-2499

10. Kim CH, Kim HK, Rettig RL, Kim J, Lee ET, Aprelikova O, Choi IJ, Munroe DJ, Green JE (2011) miRNA signature associated with outcome of gastric cancer patients following chemotherapy. BMC Med 
Genomics 4:79. doi:10.1186/1755-8794-4-79

11. Chen Y, Zuo J, Liu Y, Gao H, Liu W (2010) Inhibitory effects of miRNA-200c on chemotherapyresistance and cell proliferation of gastric cancer SGC7901/DDP cells. Chin J Cancer 29 (12):10061011. doi:10.5732/cjc.010.10236

12. Friedman J, Hastie T, Tibshirani R (2010) Regularization Paths for Generalized Linear Models via Coordinate Descent. J Stat Softw 33 (1):1-22

13. Heagerty PJ, Lumley T, Pepe MS (2000) Time-dependent ROC curves for censored survival data and a diagnostic marker. Biometrics 56 (2):337-344

14. Agarwal V, Bell GW, Nam JW, Bartel DP (2015) Predicting effective microRNA target sites in mammalian mRNAs. Elife 4. doi:10.7554/eLife.05005

15. Li JH, Liu S, Zhou H, Qu LH, Yang JH (2014) starBase v2.0: decoding miRNA-ceRNA, miRNA-ncRNA and protein-RNA interaction networks from large-scale CLIP-Seq data. Nucleic acids research 42 (Database issue):D92-97. doi:10.1093/nar/gkt1248

16. Huang da W, Sherman BT, Lempicki RA (2009) Systematic and integrative analysis of large gene lists using DAVID bioinformatics resources. Nat Protoc 4 (1):44-57. doi:10.1038/nprot.2008.211

17. Subramanian A, Tamayo P, Mootha VK, Mukherjee S, Ebert BL, Gillette MA, Paulovich A, Pomeroy SL, Golub TR, Lander ES, Mesirov JP (2005) Gene set enrichment analysis: a knowledge-based approach for interpreting genome-wide expression profiles. Proc Natl Acad Sci U S A 102 (43):15545-15550. doi:10.1073/pnas.0506580102

18. Newman AM, Liu CL, Green MR, Gentles AJ, Feng W, Xu Y, Hoang CD, Diehn M, Alizadeh AA (2015) Robust enumeration of cell subsets from tissue expression profiles. Nature methods 12 (5):453-457. doi:10.1038/nmeth.3337

19. Hanzelmann S, Castelo R, Guinney J (2013) GSVA: gene set variation analysis for microarray and RNA-seq data. BMC Bioinformatics 14:7. doi:10.1186/1471-2105-14-7

20. Singh SK, Hawkins C, Clarke ID, Squire JA, Bayani J, Hide T, Henkelman RM, Cusimano MD, Dirks PB (2004) Identification of human brain tumour initiating cells. Nature 432 (7015):396-401. doi:10.1038/nature03128

21. Visconti R, Della Monica R, Grieco D (2016) Cell cycle checkpoint in cancer: a therapeutically targetable double-edged sword. J Exp Clin Cancer Res 35 (1):153. doi:10.1186/s13046-016-0433-9

22. Thorsson V, Gibbs DL, Brown SD, Wolf D, Bortone DS, Ou Yang TH, Porta-Pardo E, Gao GF, Plaisier CL, Eddy JA, Ziv E, Culhane AC, Paull EO, Sivakumar IKA, Gentles AJ, Malhotra R, Farshidfar F, Colaprico A, Parker JS, Mose LE, Vo NS, Liu J, Liu Y, Rader J, Dhankani V, Reynolds SM, Bowlby R, Califano A, Cherniack AD, Anastassiou D, Bedognetti D, Mokrab Y, Newman AM, Rao A, Chen K, Krasnitz A, Hu H, Malta TM, Noushmehr H, Pedamallu CS, Bullman S, Ojesina Al, Lamb A, Zhou W, Shen H, Choueiri TK, Weinstein JN, Guinney J, Saltz J, Holt RA, Rabkin CS, Cancer Genome Atlas Research N, Lazar AJ, Serody JS, Demicco EG, Disis ML, Vincent BG, Shmulevich I (2018) The Immune Landscape of Cancer. Immunity 48 (4):812-830 e814. doi:10.1016/j.immuni.2018.03.023 
23. Holohan C, Van Schaeybroeck S, Longley DB, Johnston PG (2013) Cancer drug resistance: an evolving paradigm. Nat Rev Cancer 13 (10):714-726. doi:10.1038/nrc3599

24. Fojo T (2007) Multiple paths to a drug resistance phenotype: mutations, translocations, deletions and amplification of coding genes or promoter regions, epigenetic changes and microRNAs. Drug Resist Updat 10 (1-2):59-67. doi:10.1016/j.drup.2007.02.002

25. Zhao H, Zhao H, Zhang Y, Zhou Y (2018) MicroRNA199b promotes cell proliferation and invasion in Wilms' tumour by directly targeting Runtrelated transcription factor 3. Mol Med Rep 18 (2):18121819. doi:10.3892/mmr.2018.9096

26. Chen Z, Zhao G, Zhang Y, Ma Y, Ding Y, Xu N (2018) MiR-199b-5p promotes malignant progression of osteosarcoma by regulating HER2. J BUON 23 (6):1816-1824

27. Wang C, Song B, Song W, Liu J, Sun A, Wu D, Yu H, Lian J, Chen L, Han J (2011) Underexpressed microRNA-199b-5p targets hypoxia-inducible factor-1alpha in hepatocellular carcinoma and predicts prognosis of hepatocellular carcinoma patients. J Gastroenterol Hepatol 26 (11):1630-1637. doi:10.1111/j.1440-1746.2011.06758.x

28. Zhou SJ, Liu FY, Zhang AH, Liang HF, Wang Y, Ma R, Jiang YH, Sun NF (2017) MicroRNA-199b-5p attenuates TGF-beta1-induced epithelial-mesenchymal transition in hepatocellular carcinoma. $\mathrm{Br} \mathrm{J}$ Cancer 117 (2):233-244. doi:10.1038/bjc.2017.164

29. Sakaguchi T, Yoshino H, Yonemori M, Miyamoto K, Sugita S, Matsushita R, Itesako T, Tatarano S, Nakagawa M, Enokida H (2017) Regulation of ITGA3 by the dual-stranded microRNA-199 family as a potential prognostic marker in bladder cancer. Br J Cancer 116 (8):1077-1087.

doi:10.1038/bjc.2017.43

30. Fang C, Wang FB, Li Y, Zeng XT (2016) Down-regulation of miR-199b-5p is correlated with poor prognosis for breast cancer patients. Biomed Pharmacother 84:1189-1193. doi:10.1016/j.biopha.2016.10.006

31. Shen ZL, Wang B, Jiang KW, Ye CX, Cheng C, Yan YC, Zhang JZ, Yang Y, Gao ZD, Ye YJ, Wang S (2016) Downregulation of miR-199b is associated with distant metastasis in colorectal cancer via activation of SIRT1 and inhibition of CREB/KISS1 signaling. Oncotarget 7 (23):35092-35105. doi:10.18632/oncotarget.9042

32. Favreau AJ, McGlauflin RE, Duarte CW, Sathyanarayana P (2015) miR-199b, a novel tumor suppressor miRNA in acute myeloid leukemia with prognostic implications. Exp Hematol Oncol 5:4. doi:10.1186/s40164-016-0033-6

33. Wang J, Zhou F, Yin L, Zhao L, Zhang Y, Wang J (2017) MicroRNA-199b targets the regulation of ZEB1 expression to inhibit cell proliferation, migration and invasion in nonsmall cell lung cancer. Mol Med Rep 16 (4):5007-5014. doi:10.3892/mmr.2017.7195

34. Torres A, Torres K, Pesci A, Ceccaroni M, Paszkowski T, Cassandrini P, Zamboni G, Maciejewski R (2012) Deregulation of miR-100, miR-99a and miR-199b in tissues and plasma coexists with increased expression of mTOR kinase in endometrioid endometrial carcinoma. BMC Cancer 12:369. doi:10.1186/1471-2407-12-369 
35. Zhou J, Zhang Y, Qi Y, Yu D, Shao Q, Liang J (2017) MicroRNA-152 inhibits tumor cell growth by directly targeting RTKN in hepatocellular carcinoma. Oncol Rep 37 (2):1227-1234. doi:10.3892/or.2016.5290

36. Zhou X, Zhao F, Wang ZN, Song YX, Chang H, Chiang Y, Xu HM (2012) Altered expression of miR-152 and miR-148a in ovarian cancer is related to cell proliferation. Oncol Rep 27 (2):447-454. doi:10.3892/or.2011.1482

37. Chen Y, Song Y, Wang Z, Yue Z, Xu H, Xing C, Liu Z (2010) Altered expression of MiR-148a and MiR152 in gastrointestinal cancers and its clinical significance. J Gastrointest Surg 14 (7):1170-1179. doi:10.1007/s11605-010-1202-2

38. Chen H, Liu H, Zou H, Chen R, Dou Y, Sheng S, Dai S, Ai J, Melson J, Kittles RA, Pirooznia M, Liptay MJ, Borgia JA, Deng Y (2016) Evaluation of Plasma miR-21 and miR-152 as Diagnostic Biomarkers for Common Types of Human Cancers. J Cancer 7 (5):490-499. doi:10.7150/jca.12351

39. Sanfiorenzo C, llie MI, Belaid A, Barlesi F, Mouroux J, Marquette CH, Brest P, Hofman P (2013) Two panels of plasma microRNAs as non-invasive biomarkers for prediction of recurrence in resectable NSCLC. PLoS One 8 (1):e54596. doi:10.1371/journal.pone.0054596

40. Jiang X, Du L, Wang L, Li J, Liu Y, Zheng G, Qu A, Zhang X, Pan H, Yang Y, Wang C (2015) Serum microRNA expression signatures identified from genome-wide microRNA profiling serve as novel noninvasive biomarkers for diagnosis and recurrence of bladder cancer. Int J Cancer 136 (4):854862. doi:10.1002/ijc. 29041

41. Yu Y, Wu J, Guan L, Qi L, Tang Y, Ma B, Zhan J, Wang Y, Fang W, Zhang H (2013) Kindlin 2 promotes breast cancer invasion via epigenetic silencing of the microRNA200 gene family. Int J Cancer 133 (6):1368-1379. doi:10.1002/ijc.28151

42. Paterson EL, Kazenwadel J, Bert AG, Khew-Goodall Y, Ruszkiewicz A, Goodall GJ (2013) Downregulation of the miRNA-200 family at the invasive front of colorectal cancers with degraded basement membrane indicates EMT is involved in cancer progression. Neoplasia 15 (2):180-191. doi:10.1593/neo.121828

43. Soubani O, Ali AS, Logna F, Ali S, Philip PA, Sarkar FH (2012) Re-expression of miR-200 by novel approaches regulates the expression of PTEN and MT1-MMP in pancreatic cancer. Carcinogenesis 33 (8):1563-1571. doi:10.1093/carcin/bgs189

44. Bai JX, Yan B, Zhao ZN, Xiao X, Qin WW, Zhang R, Jia LT, Meng YL, Jin BQ, Fan DM, Wang T, Yang AG (2013) Tamoxifen represses miR-200 microRNAs and promotes epithelial-to-mesenchymal transition by up-regulating c-Myc in endometrial carcinoma cell lines. Endocrinology 154 (2):635-645. doi:10.1210/en.2012-1607

45. Tang H, Deng M, Tang Y, Xie X, Guo J, Kong Y, Ye F, Su Q, Xie X (2013) miR-200b and miR-200c as prognostic factors and mediators of gastric cancer cell progression. Clin Cancer Res 19 (20):56025612. doi:10.1158/1078-0432.CCR-13-1326

46. Chen HY, Lin YM, Chung HC, Lang YD, Lin CJ, Huang J, Wang WC, Lin FM, Chen Z, Huang HD, Shyy JY, Liang JT, Chen RH (2012) miR-103/107 promote metastasis of colorectal cancer by targeting the 
metastasis suppressors DAPK and KLF4. Cancer Res 72 (14):3631-3641. doi:10.1158/00085472.CAN-12-0667

47. Yu D, Zhou H, Xun Q, Xu X, Ling J, Hu Y (2012) microRNA-103 regulates the growth and invasion of endometrial cancer cells through the downregulation of tissue inhibitor of metalloproteinase 3 . Oncol Lett 3 (6):1221-1226. doi:10.3892/ol.2012.638

48. Tchernitsa O, Kasajima A, Schafer R, Kuban RJ, Ungethum U, Gyorffy B, Neumann U, Simon E, Weichert W, Ebert MP, Rocken C (2010) Systematic evaluation of the miRNA-ome and its downstream effects on mRNA expression identifies gastric cancer progression. J Pathol 222 (3):310-319. doi:10.1002/path.2759

49. Li X, Zhang Y, Zhang H, Liu X, Gong T, Li M, Sun L, Ji G, Shi Y, Han Z, Han S, Nie Y, Chen X, Zhao Q, Ding J, Wu K, Daiming F (2011) miRNA-223 promotes gastric cancer invasion and metastasis by targeting tumor suppressor EPB41L3. Mol Cancer Res 9 (7):824-833. doi:10.1158/1541-7786.MCR$10-0529$

50. Liang J, Liu X, Xue H, Qiu B, Wei B, Sun K (2015) MicroRNA-103a inhibits gastric cancer cell proliferation, migration and invasion by targeting c-Myb. Cell Prolif 48 (1):78-85. doi:10.1111/cpr.12159

51. Veldman RJ, Klappe K, Hinrichs J, Hummel I, van der Schaaf G, Sietsma H, Kok JW (2002) Altered sphingolipid metabolism in multidrug-resistant ovarian cancer cells is due to uncoupling of glycolipid biosynthesis in the Golgi apparatus. FASEB J 16 (9):1111-1113. doi:10.1096/fj.01-0863fje

52. Prinetti A, Basso L, Appierto V, Villani MG, Valsecchi M, Loberto N, Prioni S, Chigorno V, Cavadini E, Formelli F, Sonnino S (2003) Altered sphingolipid metabolism in N-(4-hydroxyphenyl)-retinamideresistant A2780 human ovarian carcinoma cells. J Biol Chem 278 (8):5574-5583. doi:10.1074/jbc.M207269200

53. Lucci A, Cho WI, Han TY, Giuliano AE, Morton DL, Cabot MC (1998) Glucosylceramide: a marker for multiple-drug resistant cancers. Anticancer Res 18 (1B):475-480

54. Fischer KR, Durrans A, Lee S, Sheng J, Li F, Wong ST, Choi H, El Rayes T, Ryu S, Troeger J, Schwabe RF, Vahdat LT, Altorki NK, Mittal V, Gao D (2015) Epithelial-to-mesenchymal transition is not required for lung metastasis but contributes to chemoresistance. Nature 527 (7579):472-476. doi:10.1038/nature15748

55. Zheng X, Carstens JL, Kim J, Scheible M, Kaye J, Sugimoto H, Wu CC, LeBleu VS, Kalluri R (2015) Epithelial-to-mesenchymal transition is dispensable for metastasis but induces chemoresistance in pancreatic cancer. Nature 527 (7579):525-530. doi:10.1038/nature16064

\section{Figures}




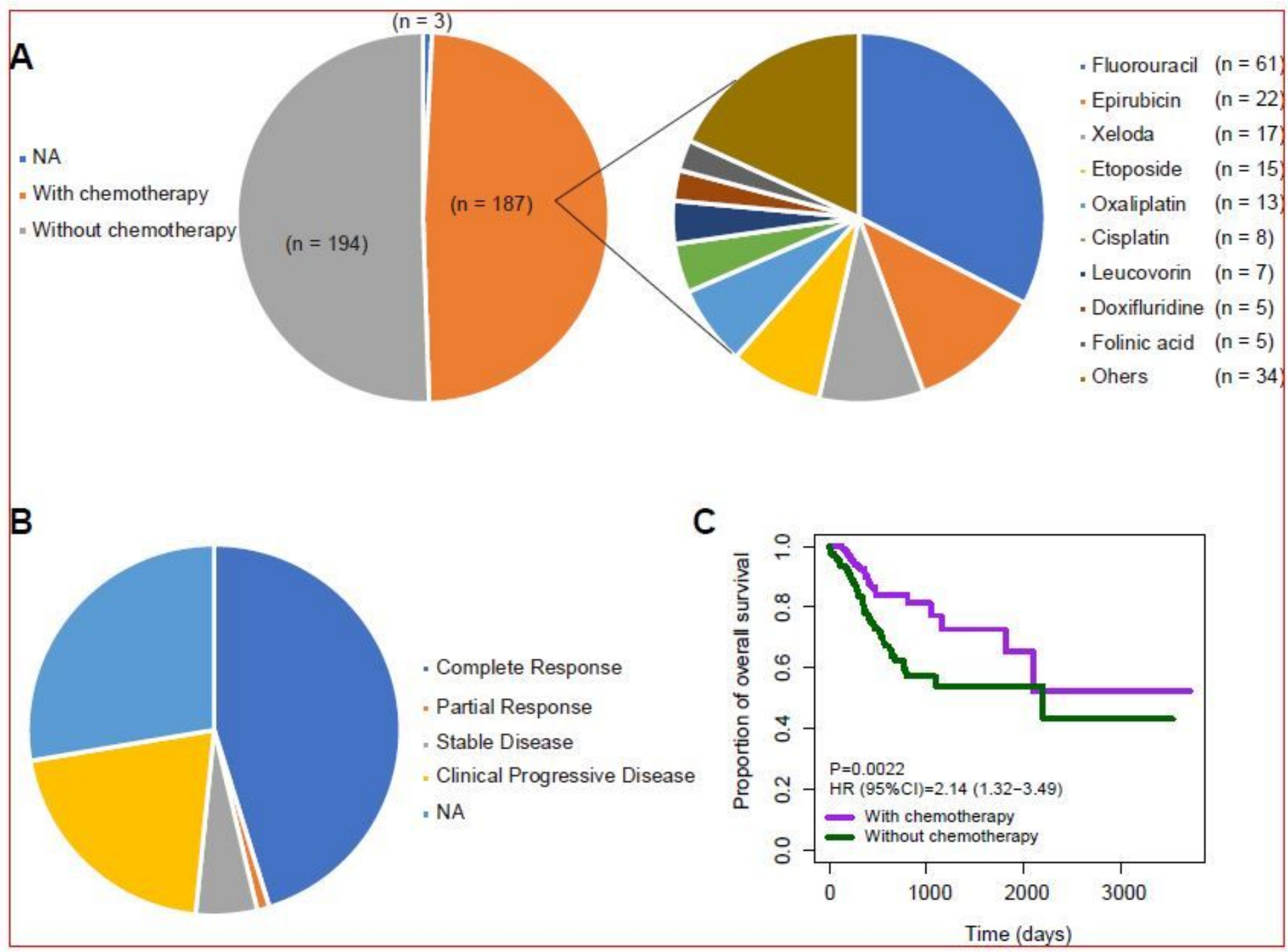

\section{Figure 1}

Adjuvant chemotherapy administration in GCs. (A) Distribution of adjuvant chemotherapy administration in GCs from the TCGA GC cohort $(n=384)$. (B) Distribution of chemotherapy response in GC patients received adjuvant chemotherapy $(n=187)$. (C) Kaplan-Meier survival curves for $G C$ patients who received adjuvant chemotherapy (GC-ACT) and those didn't receive any type of chemotherapy (GC-NOACT). 


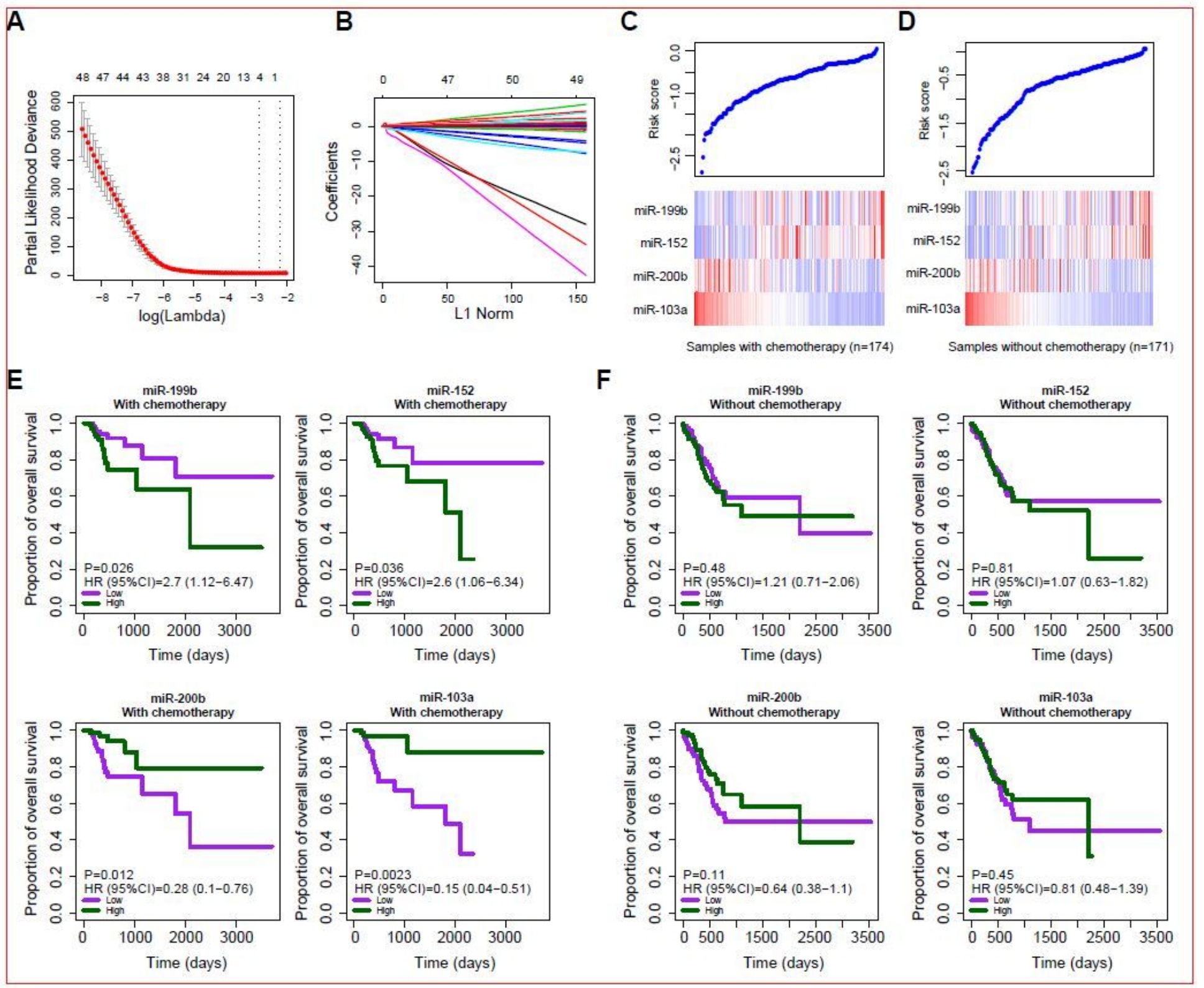

\section{Figure 2}

Identification of prognosis related miRNAs using LASSO regression model. (A) Plots of the crossvalidation error rates. Each dot represents a lambda value along with error bars to give a confidence interval for the cross-validated error rate. The top of the plot gives the size of each model. The vertical dotted line indicates the value with the minimum error and the largest lambda value where the deviance is within one SE of the minimum. (B) LASSO coefficient profiles of the miRNAs associated with the overall survival of GC patients who received adjuvant chemotherapy (GC-ACT). (C) Risk score distribution and miRNA expression profile in GC-ACT patients. Top, the distribution of each risk score for each GC-ACT patient. Bottom, heatmap of the four signature miRNAs. (D) Risk score distribution and miRNA expression profile in GC-NOACT patient. Top, the distribution of each risk score for each GC-NOACT patient. Bottom, heatmap of the four signature miRNAs. (E) Kaplan-Meier analyses the overall survival in GC-ACT patients on the basis of individual signature miRNAs. The median value of the signature miRNA expression levels as a cut-of value to divide the patients into high- and low-expression groups. (F) Kaplan-Meier analyses 
the overall survival in GC-NOACT patients on the basis of individual signature miRNAs. The median value of the signature miRNA expression levels as a cut-of value to divide the patients into high- and lowexpression groups.

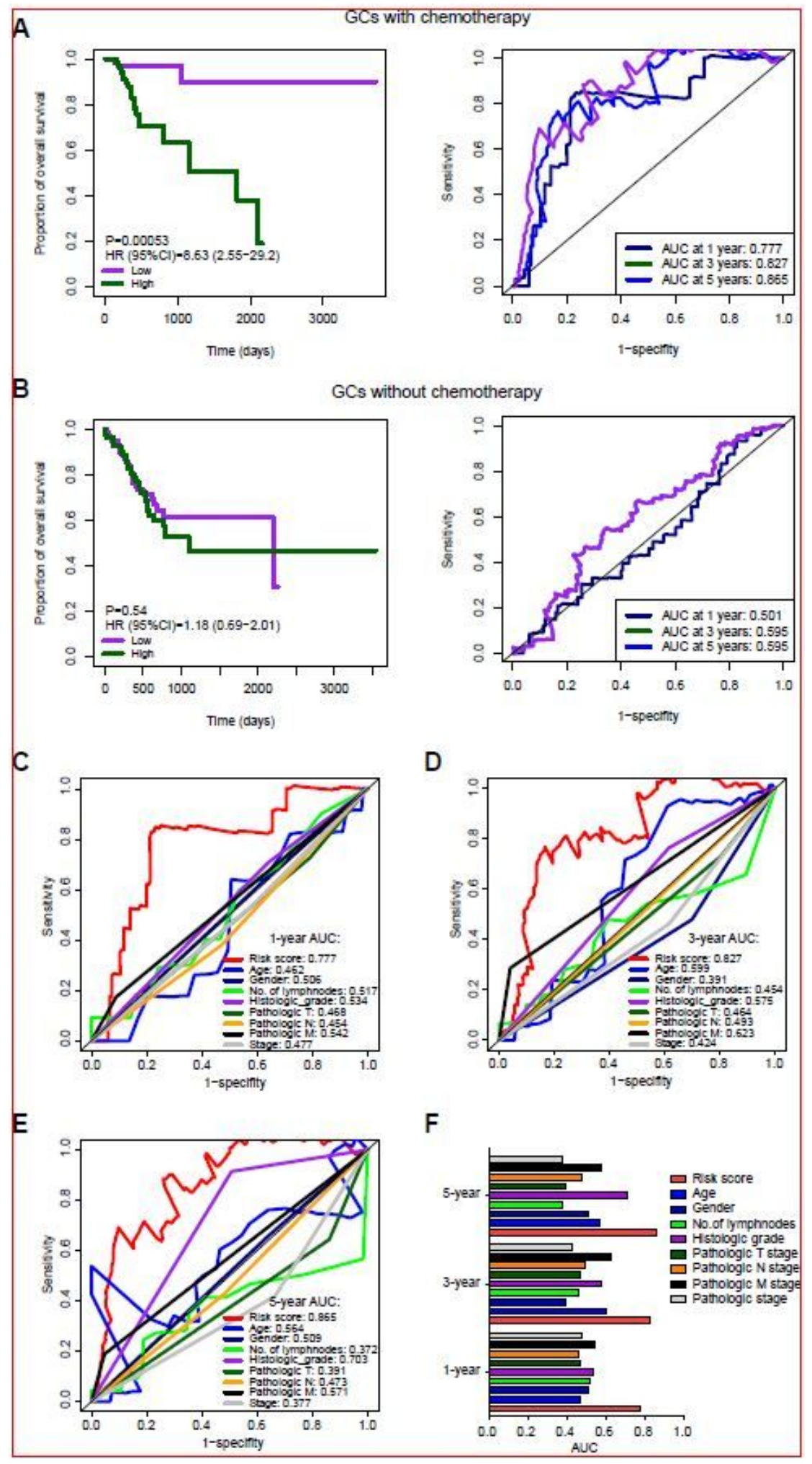

Figure 3

Survival analysis and time-dependent ROC curves for the miRNA-based prognostic signature in GCs with or without chemotherapy. (A) Left, Kaplan-Meier survival curves for four-miRNA prognostic signature in 
GC-ACT patients. Right, time-dependent ROC curves were applied to assess predictive accuracy for overall survival at varying follow-up times (1-, 3- and 5-year survival). The median value of the four-miRNA signature risk scores as a cut-of value to divide the patients into high- and low-risk groups. (B) Left, Kaplan-Meier survival curves for four-miRNA prognostic signature in GC-NOACT patients. Right, timedependent ROC curves were applied to assess predictive accuracy for overall survival at varying follow-up times (1-, 3- and 5-year survival). The median value of the four-miRNA signature risk scores as a cut-of value to divide the patients into high- and low-risk groups. (C-E) Time-dependent ROC curves for miRNAbased signature and clinicopathological risk factors for overall survival prediction at varying follow-up times (1-, 3- and 5-year survival, respectively). (F) Summary of all AUCs from (C-E). 


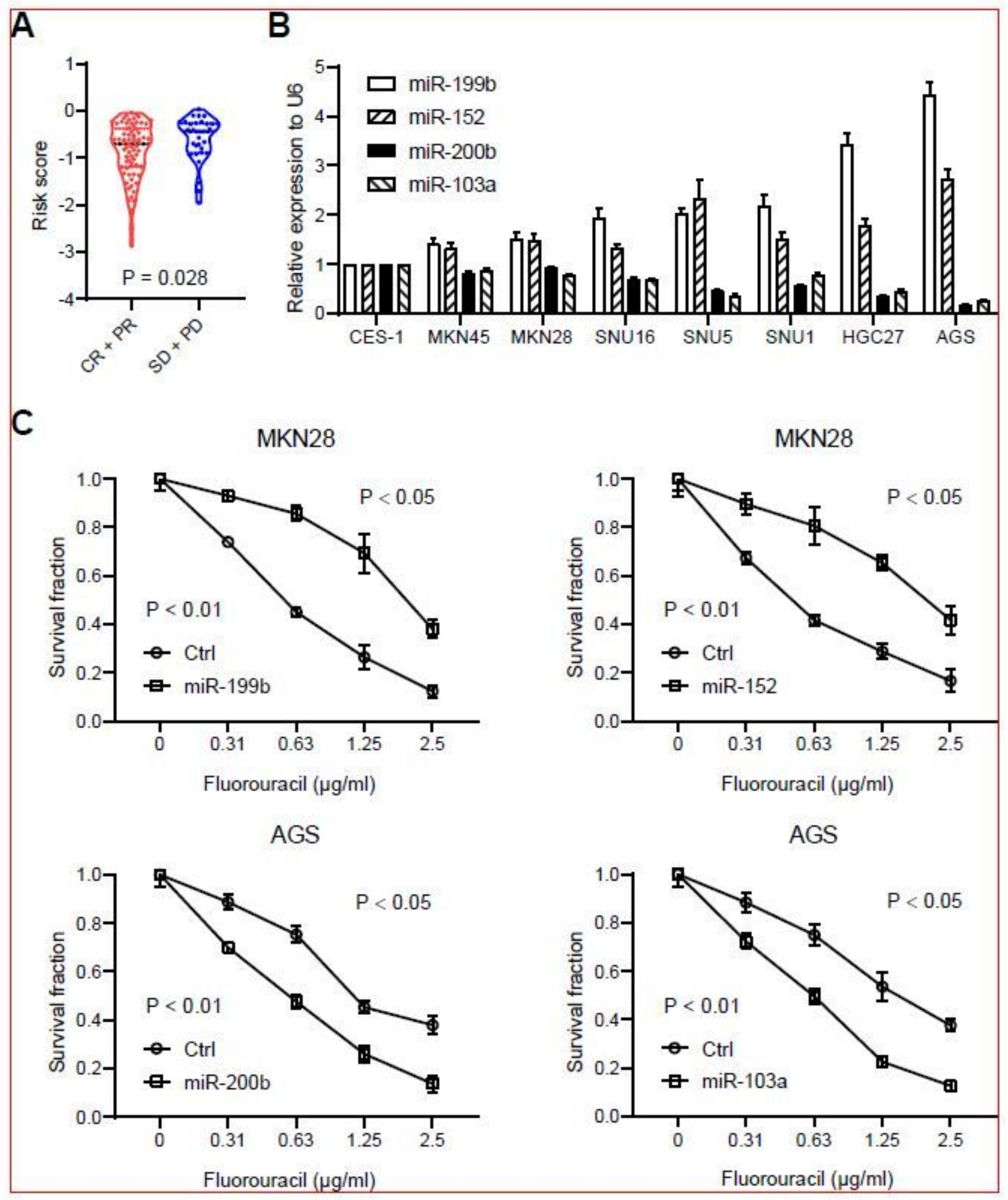

Figure 4

The functional roles of signature miRNAs in modulation of chemoresistance in GC cells. (A) Distribution of the four-miRNA signature risk scores in GC patients according to the response to chemotherapy. (B) Relative levels of signature miRNAs in a non-carcinoma CES-1 cells and a panel of GC cell lines, including MKN45, MKN28, SNU16, SNU5, SNU1, HGC27 and AGS cells. U6 was used as an internal control for normalization. (C) Top, MKN28 cells were treated with 0-2.5 $\mu \mathrm{g} / \mathrm{ml}$ 5-Fluorouracil in 96-well plates for $24 \mathrm{~h}$ 
upon overexpression of miR-199b or miR-152. Bottom, AGS cells were treated with 0-2.5 $\mu \mathrm{g} / \mathrm{ml} 5$ Fluorouracil in 96-well plates for $24 \mathrm{~h}$ upon overexpression of miR-200b or miR-103a. MTT assay was used to investigate viability of GC cells.

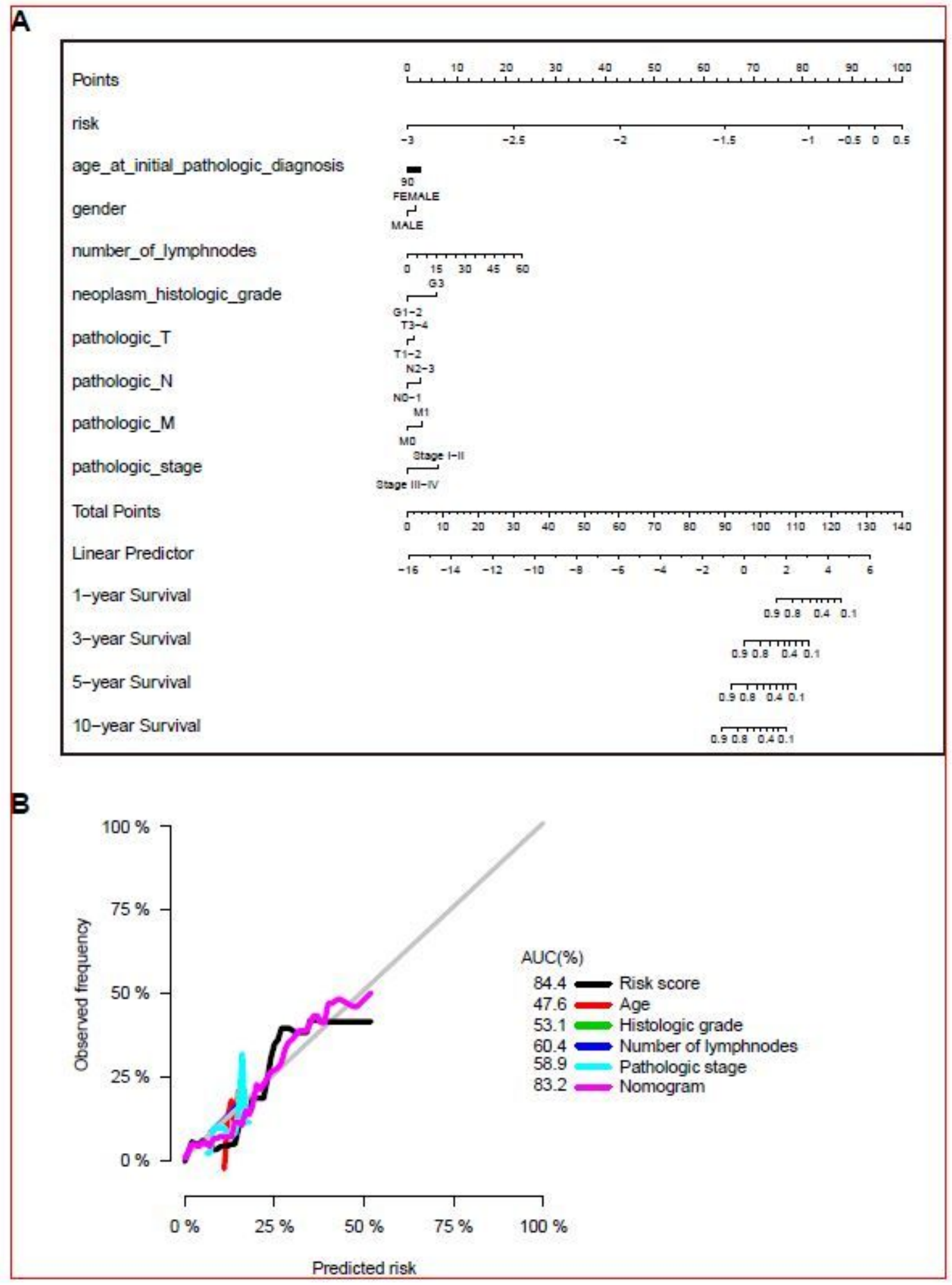

Figure 5

Establishment of the nomogram for GC patients undergoing adjuvant chemotherapy. (A) Nomogram for predicting OS of GC patients undergoing adjuvant chemotherapy. There are nine components in this 
nomogram: the four-miRNA risk score and eight clinicopathological variables. Each of them generates points according to the line drawn upward. And the total points of the three components of an individual patient lie on "Total Points" axis which corresponds to the probability of 1-, 3-, 5- and 10-year OS rate plotted on the two axes below. (B) Calibration curves for the four-miRNA proportional hazards model, clinical variable model and the combined nomogram. AUC scores are expressed as the point estimates.

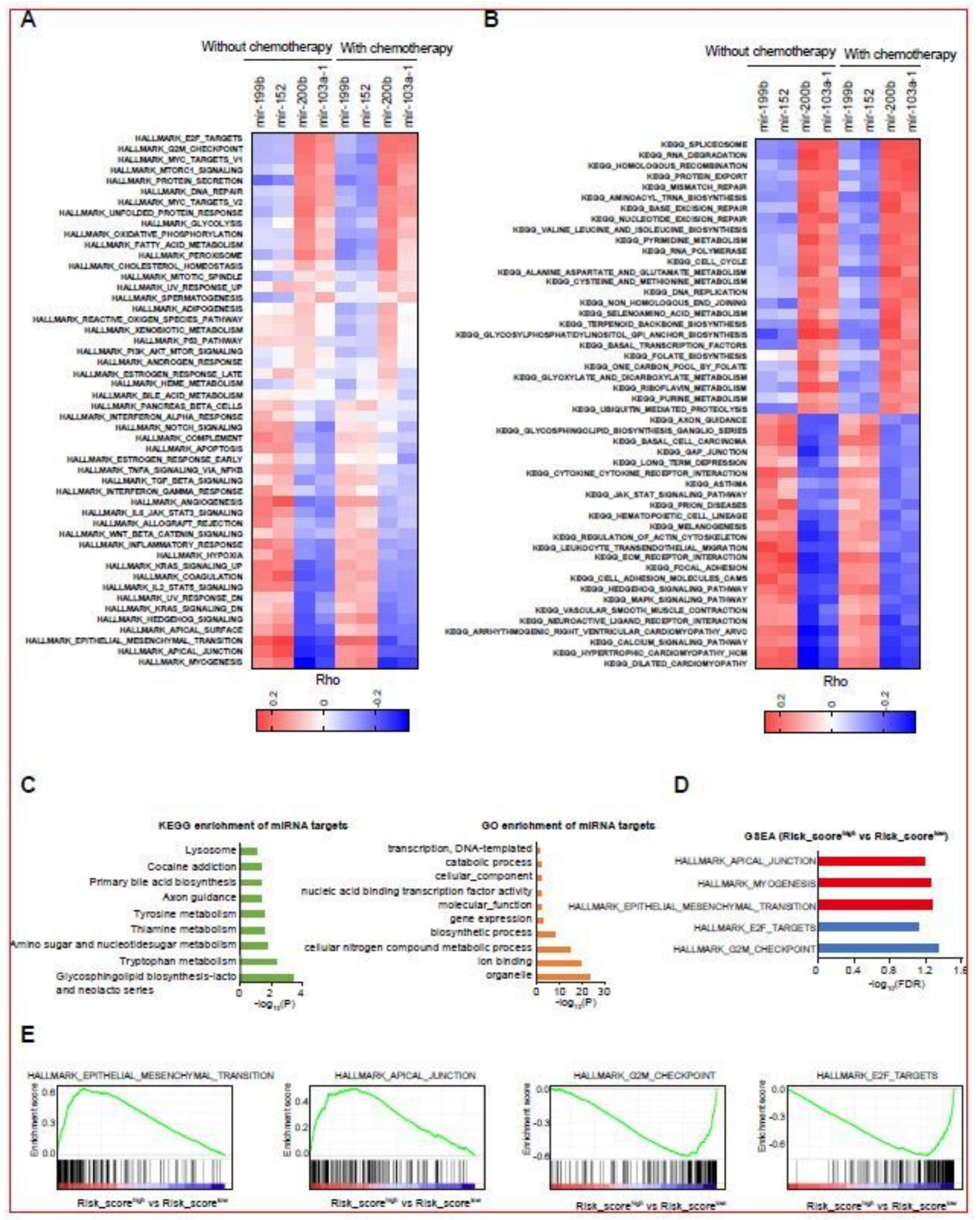

Figure 6 
Functional enrichment analyses of the signature miRNA and the miRNA-based signature (A-B) Correlations between expression levels of risk signature miRNAs and the activities of all HALLMARK pathways (A) and the top 50 significant KEGG pathways (B). The activity of pathway was inferred by single sample gene set variation analysis (GSVA) method based on the expression data of TCGA GCs. (C) KEGG pathway (left) and Gene Ontology (right) enrichment analysis for the target genes of the four signature miRNAs predicted by TargetScan and Starbase. (D-E) Gene set enrichment (GSEA) plot depicting the enrichment of hallmarks associated with the miRNA-based signature risk score. (D) Significant hallmarks enriched in high-risk GC-ACT patients (red) or low-risk GC-ACT patients (blue) (FDR $<0.1$ ). (E) GSEA plots showing the representative enriched hallmarks in high-risk GC-ACT patients (top) or low-risk GC-ACT patients (bottom). 


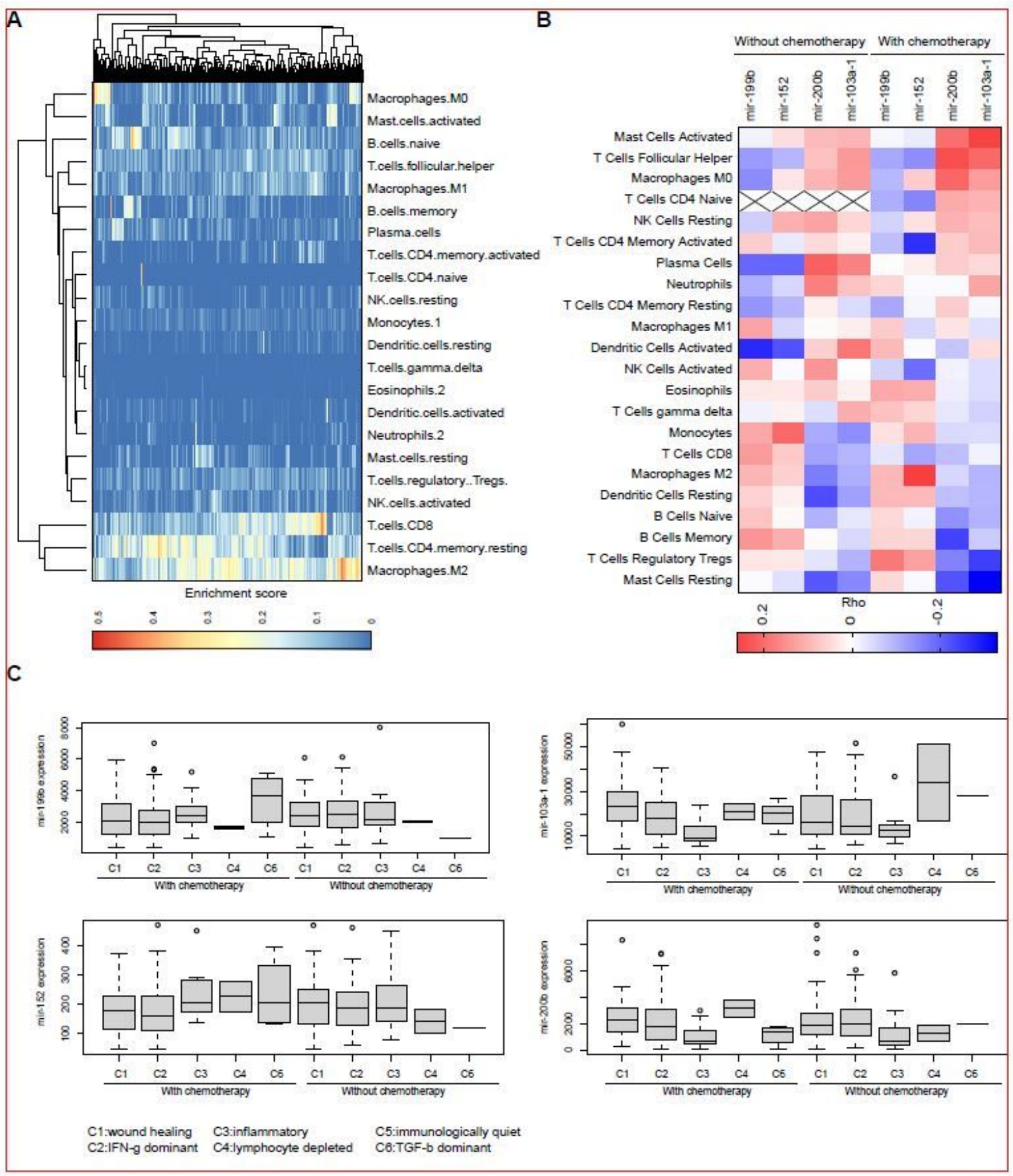

Figure 7

Risk signature miRNAs-immune cell associations in GCs (A) Heatmap of immune cell populations in GC tumor tissues inferred by CIBERSORT cellular composition analysis. (B) Heatmap of correlation between the immune cell populations and expression levels of four risk signature miRNAs in GC tumors. The correlation strength was assessed by Spearman's rank correlation analyses. (C) The expression levels of 
four risk signature miRNAs in GC tumors grouped according to the immune subtypes, including $\mathrm{C} 1$-wound healing, C2-IFN- $\gamma$ dominant, C3-inflammatory, C4-lymphocyte depleted and C6-TGF- $\beta$ dominant.

\section{Supplementary Files}

This is a list of supplementary files associated with this preprint. Click to download.

- Supplementarytables.docx 